Militaires et sécurité intérieure.

\title{
Editorial - Militaires et sécurité intérieure : l'Irlande du Nord comme métaphore
}

\author{
Didier Bigo et Emmanuel-Pierre Guittet
}

\section{(2) OpenEdition \\ 1 Journals}

\section{Édition électronique}

URL : http://journals.openedition.org/conflits/1607

DOI : 10.4000/conflits. 1607

ISSN : $1777-5345$

Éditeur :

CCLS - Centre d'études sur les conflits lilberté et sécurité, L'Harmattan

\section{Édition imprimée}

Date de publication : 1 décembre 2004

Pagination : 5-10

ISBN : 2-7475-7598-X

ISSN : 1157-996X

\section{Référence électronique}

Didier Bigo et Emmanuel-Pierre Guittet, « Editorial - Militaires et sécurité intérieure : I'Irlande du Nord comme métaphore », Cultures \& Conflits [En ligne], 56 | hiver 2004, mis en ligne le 07 janvier 2010, consulté le 30 mars 2021. URL : http://journals.openedition.org/conflits/1607 ; DOI : https://doi.org/ 10.4000/conflits. 1607

Ce document a été généré automatiquement le 30 mars 2021.

Creative Commons License 


\title{
Editorial - Militaires et sécurité intérieure : l'Irlande du Nord comme métaphore
}

\author{
Didier Bigo et Emmanuel-Pierre Guittet
}

1 Ce nouveau numéro de Cultures \& Conflits sur « Militaires et sécurité intérieure. L'Irlande du Nord comme métaphore " poursuit la réflexion sur la participation des militaires à la sécurité intérieure entamée dans le numéro 44 de la revue sous le titre "Défense et identités. Un contexte sécuritaire global ? ». Il analyse les moments où les militaires veulent faire retour sur l'interne et la protection des frontières, en les corrélant aux discours des professionnels de la politique tirant argument d'une menace existentielle pour justifier des mesures dérogatoires au droit des individus, une restriction des libertés, voire un état d'urgence ou un état d'exception. Il approfondit ce que nous avions publié à l'hiver 2001, en réponse aux thèses de l'hyper-terrorisme, de la supposée nouveauté incommensurable des attentats du 11 septembre 2001. Nous avions alors signalé les discours d'euphémisation savante des docteurs Pangloss refusant d'admettre un quelconque changement et les discours inverses des prophètes de peur justifiant, au nom de la démocratie en péril et d'un futur dangereux, la nécessité d'employer des moyens à la mesure du danger, et par là même, d'écarter toute démarche judiciaire au profit d'une voie militaire ${ }^{1}$.

2 Cette question de la participation des militaires à ce qui a été appelé la sécurité intérieure pour évoquer les relations entre polices, services de renseignement et armée va bien plus loin que le discours technico-institutionnel de la collaboration civilomilitaire ne le prétend. Elle trouve dans l'Irlande du Nord une de ses illustrations les plus riches d'enseignement, surtout lorsqu'on la croise avec les discours politiques sur la nécessité de restreindre temporairement les libertés individuelles et l'accès à la justice. Et c'est pourquoi nous proposons un retour réflexif sur l'Irlande du Nord afin d'éclairer différemment les discours contemporains sur l'état d'urgence après le 11 septembre 2001. L'Irlande du Nord nous sert de métaphore pour comprendre la relation entre discours d'exception et pratiques militaires de surveillance et de contrôle qui 
importent à l'intérieur même du pays un climat de guerre. Beaucoup d'ouvrages ont analysé cette collaboration entre police et armée à travers une réflexion plus large sur la décolonisation, sur les conflits intercommunautaires, sur les luttes entre factions religieuses ou sur la lutte contre les terroristes et extrémistes de tout bord ainsi que sur les équilibres liberté sécurité dans un contexte intercommunautaire qui supposerait l'arbitrage d'un tiers parti. Ici, nous précisons la focale, et prenons cette « collaboration des militaires au pouvoir civil » comme axe central de la recherche, afin d'en comprendre les logiques propres et le rapport avec les pratiques de la lutte « antisubversive ». Tel est le sens de la publication d'un extrait du rapport établi par Didier Bigo, Emmanuel-Pierre Guittet et Andy Smith². En Irlande du Nord, l'armée britannique, obéissant au pouvoir politique de Londres, et qui s'en tient à ce discours parfaitement officiel d'une aide aux pouvoirs civils, a développé toute une doctrine interne d'emploi des forces qui s'apparente à la lutte antisubversive et à l'expérience de la décolonisation en ayant recours à l'argument de la multiplicité des pratiques pouvant s'inscrire dans le cadre de la mission militaire ("guerre urbaine», "conflit de basse intensité ", " contrôle des foules ", " maîtrise de la violence »...). En étudiant le manuel d'emploi des forces dans le contrôle des foules de l'armée de Terre française, Elwis Potier montre que ces modes d'appréhension de la gestion de la violence, ne sont pas spécifiques aux britanniques et que le discours doctrinal français singulier de la «maitrise de la violence» obéit aux mêmes logiques de fonctionnement. Si la préoccupation des militaires pour la sécurité intérieure est souvent reliée aussi à un discours politique sur la nécessité de l'état d'urgence ou d'une situation d'exception supposant une menace attentant à la survie même du pays, les gouvernements s'imposent souvent toutefois des limites dans le temps ou dans l'espace, ou dans l'objet de l'exception. En Italie, c'est l'expérience Vespri Siciliani relatée par Anastassia Tsoukala, et le cadre de la lutte contre la drogue et les mafias, en France, c'est le cadre de la lutte antiterroriste et le plan vigipirate qui s'est installé dans la vie quotidienne. Mais l'exception aux règles de droit et le contrôle par les militaires de la vie civile n'ont jamais été aussi poussés en termes de reconduite indéfinie, que dans le cadre britannique de la gestion des «troubles » en Irlande du Nord, même si spatialement la configuration restait spécifique. Le résultat en a été que pendant longtemps l'Angleterre, l'Ecosse et le Pays de Galles ont vécu une situation radicalement différente au quotidien en terme de libertés publiques de celle de l'Irlande du nord et cela a produit un effet non voulu par ceux qui avaient instauré cette logique d'exception, à savoir détruire peu à peu le sentiment d'unité de l'identité britannique. L'échec de l'expérience a néanmoins toujours été présenté par les autorités comme un succès. Et, dès lors, on a voulu s'en servir comme exemple à suivre pour la lutte antiterroriste et pour justifier le rôle des militaires au sein de cette lutte. Le transfert de certaines mesures prises contre l'IRA vers les organisations clandestines du Moyen Orient qui ont voulu frapper à Londres, ainsi que vers les manifestations kurdes de protestation, font que les mesures antiterroristes récentes en Grande-Bretagne sont, pour les territoires anglais et gallois, parmi les plus sécuritaires de tous les pays européens et ce, dès avant le 11 Septembre $2001^{3}$. On a donc importé en bloc en Grande-Bretagne les procédures d'exception nord- irlandaise en les considérant comme normales et efficaces. On a élargi les procédures en termes d'espace, d'objet et de temporalité. Le 11 Septembre 2001 a été à cet égard un catalyseur, justifiant a posteriori des mesures prises précédemment, et arguant pour de nouvelles. 
3 La récente condamnation, en décembre 2004, par la plus haute instance judiciaire britannique de la législation antiterroriste permettant la détention illimitée ${ }^{4}$, sans accès des étrangers soupçonnés d'activité terroriste à leurs dossiers, marque-t-elle un tournant et le retour à des limitations acceptées par tous? Cet arrêt prononcé par les Law Lords n'a pas valeur juridique contraignante et les récents propos du nouveau ministre de l'Intérieur, Charles Clarke sur le Control Order, invitent à la circonspection, même s'il a bien été obligé de tenir compte de ce coup de semonce ${ }^{5}$. En effet les Law Lords peuvent s'appuyer sur le Human Rights Act et sur la juridiction de la Cour Européenne des Droits de l'Homme pour mettre en difficulté le gouvernement qui n'a plus le « dernier mot » sur la définition de la situation comme situation d'exception ${ }^{6}$.

Maître Nuala Mole, dans son entretien, fait le tour des récentes procédures engagées contre les militaires britanniques en poste en Irlande du Nord et revient sur ce point central des décisions de la Cour Européenne des Droits de l'Homme dévoilant combien le discours d'exception sur l'Irlande du Nord joué par les britanniques, visait à justifier l'usage de pratiques militaires sur le territoire britannique à travers la mise en place de législations spéciales dérogatoires au droit commun: "Special Powers Act", lois permettant internements, arrestations et couvre-feux. Le droit de la dérogation est alors enjeu de lutte entre l'exécutif et le législatif qui veulent en faire un moyen de mettre en place des politiques répressives qui font la part belle aux différents services de sécurité ${ }^{7}$ et les juges qui exigent de vérifier la proportionnalité des actions. Cette expérience nord-irlandaise nous renvoie parfaitement à ce qui fut cette logique de subordination du processus judiciaire national aux impératifs de sécurité où la mise en place de mesures dérogatoires entraîne quasi automatiquement ce que l'on nomme, toujours a posteriori, des « dérives ", et aux luttes qui s'en suivent à l'échelle bilatérale (Irlande/Royaume-Uni) et Européenne.

5 Sur ce point, l'exemple de ce qui s'est produit à Guantanamo, bien que différent de Belmarch, pose les mêmes questions de fond sur les relations entre exécutif et judiciaire dans un contexte favorisant l'unanimisme politique et la restriction des libertés publiques, et les relations entre la souveraineté d'un pays, sa projection à l'extérieur et une Europe qui se constitue judiciairement comme espace transnational se voulant soumis à l'Etat de Droit. Maître William Bourdon, avocat des détenus français à Guantanamo-Bay, nous explique les enjeux de cet espace proprement ajuridique de Guantanamo résultant de l'ambiguïté de la notion d' "unlawful combattant " et de la guerre contre le terrorisme, et les possibilités ouvertes par le droit européen et international pour interpeller les gouvernants américains sur leurs pratiques.

6 La critique utile des formes d'arbitraire gouvernemental ne signifie pas à l'inverse de dire que tout est comme avant, qu'il n'y a pas de violence. Des réglementations sont nécessaires dans des domaines spécifiques. Elles peuvent être prises dans un cadre légal normal et par des négociations internationales. Les mesures de sécurité aérienne que décrit Yann Poincignon renforceront sans doute à juste titre la difficulté de commettre des attentats par les mêmes moyens, mais au prix d'intrusions importantes dans la vie privée des personnes et des compagnies ${ }^{8}$. Utiles, elles sont néanmoins instrumentalisées dans des jeux bureaucratiques et politiques internationaux. La réévaluation du danger, nécessaire dans la prise en compte des modes d'action des auteurs des attentats, en matière de sécurité aérienne, de transport n'a-t-elle pas été soumise à l'intérêt d'une assignation américaine unilatérale de mesures restrictives à l'encontre de la politique européenne? 
7 Jean-Paul Hanon montre très clairement que l'implication des militaires dans la lutte antiterroriste au nom de l'efficacité est, à long terme, contre-productive ainsi que le montre, autrefois le cas algérien, maintenant le cas irakien. L'image que l'on se fait du futur aux Etats-Unis, lu comme un possible Armaggedon, fait que toute mesure préventive est de facto proportionnée puisqu'elle est censée empêcher l'apocalypse. Le scénario du pire est un scénario paranoïaque. L'Europe doit-elle le partager? Nous ne connaissons pas le futur et nous ne pouvons pas le prédire ${ }^{9}$. En discutant notamment le National Strategy Act à la lumière du texte de Philip K. Dick, Francesco Ragazzi dessine les contours de cet argument de prévention qui se joue de la forme énigmatique de la terreur. La question centrale que nous devons discuter est bien celle là. La nordirlandisation du monde où les Etats-Unis font peser, encore plus sur les autres que sur eux-mêmes, le poids de l'état d'exception, comme l'Angleterre l'a fait avec l'Irlande du Nord, ne débouche-t-elle pas sur une police militaire globale, comme le propose Alessandro dal Lago ? Il $\mathrm{n}$ y a certes pas militarisation du politique au sens classique du terme où les militaires prennent le pouvoir comme en Amérique latine dans les années 1970. Il n'y a pas non plus une militarisation technique de la vie civile avec des patrouilles militaires dans les rues sauf sans doute dans le cas de Vigipirate. En revanche, il y a bien utilisation des technologies de surveillance aux mains des militaires, en particulier les satellites d'écoute et de surveillance, et par ailleurs avec le military order il y a une tentative de militarisation de la justice qui va de pair avec l'établissement d'une police de renseignement fondé sur la suspicion et la mise à l'écart d'une police judiciaire fondée sur la recherche des preuves du crime. Cette police globale développe les technologies de surveillance de manière interne et transnationale d'une part, les actions militaires guerrières d'autre part. Elle n'est ni armée, ni police classique. Elle n'est pas non plus une gendarmerie du monde au sens d'un pouvoir impérial se déployant sans frontières. Mais elle ressemble, à une autre échelle, à ce que les pratiques britanniques en Irlande du Nord nous ont enseigné, avec les mêmes illusions momentanées des discours sécuritaires et des institutions de coercition sur leur succès, et l'obligation de les abandonner in fine pour des logiques politiques de médiation, seules capable de réinstaurer, sinon la paix, du moins le cessez-le-feu. Un Good Friday global est-il possible? L'Irlande du Nord nous offre donc par ses contradictions et ses problèmes tant locaux qu'européens, la métaphore du monde contemporain. Avec les événements du 11 septembre 2001 et la volonté à l'échelle internationale cette fois de développer des législations d'exception s'harmonisant sur les normes les plus sécuritaires indépendamment des trajectoires historiques des Etats et de leur équilibre interne sécurité/liberté, peut-on parler de nord-irlandisation du monde ${ }^{10}$ ?

\section{NOTES}

1. Bigo D., « La voie militaire de la guerre au terrorisme », Cultures \& Conflits, $n^{\circ} 44$, hiver $2001, \mathrm{pp}$. 5-18. Voir aussi Bigo D., Guittet E.-P., " éditorial - Facettes de l'insécurité », Cultures \& Conflits, n'51, automne 2003, pp. 5-7. 
2. Bigo D., Guittet E.-P., Smith A., «La participation des militaires à la sécurité intérieure : Royaume-Uni, Irlande du Nord », Rapport DAS, Juillet 2001.

3. Le Terrorism Act de juillet 2000 est une réforme et un élargissement des législations antiterroristes britannique antérieures, à savoir : le Prevention of Terrorism (Temporary Provisions) Act de 1989, le Northern Ireland (Emergency Provisions) Act de 1996 et le Criminal Justice (Terrorism and Conspiracy) Act de1998.

4. Suivant la section 23 de la loi antiterroriste 2000, rajoutée après les attentats du 11 septembre 2001. Sur la déclaration des Law Lords, voir Guild E., «Constitutional Crisis in the UK over Detention of Foreigners ", http://www.libertysecurity.org/article83.html

5. Voir la réponse de Charles Clarke, House of Commons, «Hansard Debates », 26 janvier 2005. Sur le sujet, voir CHALLENGE, http://www.libertysecurity.org

6. Voir Guild E., "Agamben face aux juges. Souveraineté, exception et antiterrorisme", Cultures\&Conflits, $\mathrm{n}^{\circ} 51$, automne 2003, pp. 127-156.

7. Walsh D., Bloody Sunday and the rule of Law in Northern Ireland, London, Macmillan Press, 2000.

8. Sur le sujet, voir l'article de Valsamis Mitsilegas sur les Passenger National Record (PNR) et l'accord d'échanges de données signé entre l'Union européenne et les Etats-Unis, à paraître dans un prochain numéro. Voir aussi Bonditti P., «From Territorial Space to Networks : A Foucaldian Approach to the Implementation of Biometry ", Alternatives, n²9, 2004, pp. 465-482.

9. Bigo D., "The globalisation of (in)security", Traces, London/Beijin, mars 2004 ; et Bigo D., Policing Insecurity today, London, Palgrave (à paraître).

10. Cet aspect est évoqué par Bigo D., « Reassuring and Protecting: Internal Security Implications of French Participation in the Coalition against Terrorism », in, Hershberg E., Moore K. W., Critical views of September 11: analyses from around the world, New-York, Norton, 2002.

\section{INDEX}

Mots-clés : Editorial, militaires, sécurité intérieure, Irlande du Nord, Royaume-Uni

\section{AUTEURS}

\section{DIDIER BIGO}

Didier BIGO est Maître de Conférences des Universités à l'IEP de Paris. Chercheur associé au Centre d'Etudes et de Recherches Internationales (CERI) et Président du Centre d'Etudes sur les Conflits, il dirige les programmes cadres de recherche et développement européens ELISE (European Liberty and Security, PCRD5) et CHALLENGE (Changing the Landscape of European Security, PCRD6).

\section{EMMANUEL-PIERRE GUITTET}

Emmanuel-Pierre GUITTET est doctorant en sociologie politique au Laboratoire d'Analyses des Systèmes Politiques (LASP), Université Paris X-Nanterre, chargé de recherches au Centre d'Etudes sur les Conflits, il est aussi Junior Researcher dans les programmes ELISE et CHALLENGE (5ème et 6ème PCRD de la Commission européenne). 